\title{
Pharmacogenomic biomarkers as inclusion criteria in clinical trials of oncology-targeted drugs: a mapping of ClinicalTrials.gov
}

\author{
Alexandre Vivot, MD, MPH ${ }^{1,2}$, Jacques Li, MD ${ }^{1,2}$, Jean-David Zeitoun, MD, MHPM ${ }^{1,2}$, Samia Mourah, MD, PhD ${ }^{3,4}$, \\ Perrine Crequit, MD, MSc ${ }^{1,2}$, Philippe Ravaud, MD, $\mathrm{PhD}^{1,2,5,6}$ and Raphaël Porcher, $\mathrm{PhD}^{1,2,5}$
}

Purpose: The aim of this study was to describe pharmacogenomicsbased inclusion criteria (enrichment) and the main characteristics of clinical trials involving oncology-targeted therapies.

Methods: Clinical trials of oncology-targeted therapies approved after 2005 with pharmacogenomic testing required or recommended in their label were retrieved from a mapping of the ClinicalTrials.gov database.

Results: We examined information for 12 drugs and 858 trials. Overall, 434 trials (51\%) were enriched on the biomarker first mentioned in the label and $145(17 \%)$ were enriched on another biomarker, whereas 270 trials (31\%) included all patients. The median proportion of trials corresponding to both the drug's indication and drug's target was $35 \%$. Of the 361 trials that tested drugs in another disease than the first one in the label, 219 (61\%) were without enrichment and $87(24 \%)$ were actually enriched but on another biomarker than the first one in the label.

Conclusion: Several drugs have been tested in trials enriched on many different biomarkers. Nonetheless, most targeted therapies have been developed only using biomarker-positive patients; therefore, exclusion of biomarker-negative patients from treatment relies on only preclinical data and on biological understanding of the disease and target.

Genet Med advance online publication 17 December 2015

Key Words: biomarkers; clinical trials; Food and Drug Administration; personalized medicine; pharmacogenomics

\section{INTRODUCTION}

In recent years oncology care has been completely transformed by the development and growth of molecular targeted therapies, ${ }^{1}$ which has led to the concept of precision (or personalized or genomic) oncology. ${ }^{2,3} \mathrm{~A}$ landmark example is imatinib, which has revolutionized the treatment of chronic myeloid leukemia. Other examples are vemurafenib for melanoma, trastuzumab and ado-trastuzumab for breast cancer, and crizotinib for lung cancer. These drugs need a pharmacogenomic biomarker to guide treatment decision making ${ }^{4}$ because the indications for these drugs are based on biomarkers (e.g., unresectable or metastatic melanoma with BRAF V600E mutation for vemurafenib). However, some targeted therapies, such as bevacizumab, do not require a biomarker assessment before prescription. This does not mean that no pharmacogenomic biomarker actually exists, but rather that none has yet been validated or even found. Furthermore, some drugs, such as everolimus, may have one biomarker-based indication (advanced hormone receptor-positive, HER-2negative breast cancer) and another non-biomarker-based indication (advanced renal cell carcinoma). Currently, the labels of 140 US Food and Drug Administration (FDA)-approved drugs mention a pharmacogenomic biomarker, one-third of which are indicated in oncology. ${ }^{5}$

A drug that has been tested only in a biomarker-positive subpopulation would have an implicit requirement for genetic testing, ${ }^{5,6}$ and one may consider that a high-quality trial with only biomarker-positive patients (i.e., an enriched trial) corresponds with an acceptable level of evidence for targeted therapies. ${ }^{7,8}$ Nonetheless, if no clinical data are available for biomarker-negative patients, the use of a biomarker to restrict treatment to a subgroup of patients may not be relevant, as noted by several regulatory agencies. ${ }^{69,10}$ It may seem natural and logical to study a targeted drug only in patients with tumors harboring this target; the hallmark trial design for targeted therapy is thereby an enriched design. This design increases the study power and therefore reduces the sample size and time to detect an effect, ${ }^{11,12}$ but it does not provide information about the drug's efficacy in biomarker-negative patients. Actually, considering the treatment effect to be null in biomarker-negative patients a priori means withdrawing treatment for some patients based on only preclinical data and the biological understanding of the drug's mechanism of action and its target. Therefore, if the biomarker is not a perfect predictor of response (because of the

${ }^{1}$ Assistance Publique des Hôpitaux de Paris (AP-HP), Hôpital Hôtel Dieu, Centre d’Épidémiologie Clinique, Paris, France; ${ }^{2}$ INSERM, UMR1153 Epidemiology and Biostatistics Sorbonne Paris Cité Research Center (CRESS), METHODS Team, Paris Descartes University, Paris, France; ${ }^{3}$ Department of Pharmacology-Genetics, Assistance Publique des Hôpitaux de Paris (AP-HP), Saint-Louis Hospital, Paris, France; ${ }^{4}$ INSERM UMRS 976, Paris, France; ${ }^{5}$ Faculté de Médecine, Université Paris Descartes, Paris, France; ${ }^{6}$ Department of Epidemiology, Mailman School of Public Health, Columbia University, New York, New York, USA. Correspondence: Alexandre Vivot (alexandre.vivot@aphp.fr)

Submitted 30 July 2015; accepted 5 October 2015; advance online publication 17 December 2015. doi:10.1038/gim.2015.165 
test's intrinsic qualities or because the treatment is also effective in some true-negative patients), one may deny patients a treatment they would have otherwise benefited from. ${ }^{9,13,14}$

We have several examples of this risk of denying treatment to some potential patients. For example, some patients with HER2negative tumors have benefited from trastuzumab. ${ }^{15}$ Indeed, 16 of 22 HER-2-negative patients with an HER-2 extracellular domain level $>15 \mathrm{ng} / \mathrm{mL}$ showed a clinical benefit, which led to the concept of "hidden" HER-2-positive breast cancer. ${ }^{16}$ Furthermore, contrary to expectations, the level of HER-2 did not predict clinical response and showed a $U$-shaped relationship, which suggested that trastuzumab could be effective in HER-2-negative tumors. ${ }^{17}$ Another example is cetuximab, the anti-epidermal growth factor receptor (EGFR) drug, originally studied only in patients with EGFR-expressing tumors until it was found to be beneficial for some patients with EGFR negative tumors as well. ${ }^{18,19}$ Later, retrospective analyses of randomized clinical trial data revealed that only patients with $R A S$ wild-type tumors benefited from the treatment. ${ }^{20,21}$ The anti-EGFR therapies cetuximab and panitumumab are now restricted to patients with $R A S$ wild-type tumors.

The emergence of targeted therapies has also transformed drug development. ${ }^{19,22-25}$ The ideal goal in personalized medicine is the co-development of the drug and the biomarker (i.e., the biomarker is found in the early stages of drug development, and both the drug and the biomarker test are developed concurrently). Nonetheless, for some drugs, the biomarker could have been discovered after the drug was approved, and for other drugs, supplemental biomarkers could have been discovered after the discovery of the first one; hence, a targeted drug could have several pharmacogenomic biomarkers. Both drug-target development and characteristics of clinical trials evaluating targeted therapies-especially pharmacogenomic biomarkers used as inclusion criteria-are of crucial importance to understanding how those drugs are studied.

We aimed to map the clinical trial development for oncologytargeted therapies in order to assess the main characteristics of clinical trials involving oncology-targeted therapies and their evolution after first FDA approval. We analyzed (i) the characteristics of oncology-targeted therapies with pharmacogenomic testing required or recommended on their label; (ii) the characteristics of corresponding registered clinical trials, especially the use of an enrichment design; and (iii) the evolution of these characteristics over the drug development period.

\section{MATERIALS AND METHODS}

\section{List of drugs}

On 10 November 2014, we extracted the list of oncology-targeted therapies from the US National Cancer Institute website. ${ }^{26}$ We then selected from the FDA Table of Pharmacogenomic Biomarkers in Drug Labeling ${ }^{27}$ drugs for which the label required or recommended genetic testing, based on a previous work. ${ }^{5}$ We excluded drugs approved before 2005 because the International Committee of Medical Journal Editors required prospective registration of clinical trials as a precondition for publication in September 2004 (ref. 28). For each included drug, we searched ClinicalTrials.gov on 10 November 2014 using the name of the drug as a keyword, and we extracted all retrieved studies without any additional limits.

\section{Eligibility of clinical trials}

Clinical trials were included if they were interventional clinical trials evaluating one included targeted drug as an experimental treatment (alone or combined with another drug). Two authors (A.V. and J.L.) independently assessed eligibility; any disagreements were resolved by consensus.

\section{Data extraction}

Two authors (A.V. and J.L.) independently extracted data on biomarker-based criteria for including patients in clinical trials (i.e., enrichment) and, when appropriate, the names of the biomarkers. They compared all the extracted data, and any disagreements were resolved by consensus. We considered studies of chronic myelogenous leukemia, a disease associated with Philadelphia chromosome, as being implicitly enriched on this biomarker because Philadelphia chromosome is part of this disease's definition. ${ }^{29}$ Other trial characteristics were extracted from ClinicalTrials.gov. The primary outcome was coded as overall survival or event-free survival (progressionfree, disease-free, or any event-free survival as defined in the trial registration), or time to progression, following this hierarchical and exclusive classification. To classify the diseases studied in each trial, we mapped the Medical Subject Heading terms from ClinicalTrials.gov records to the list of diseases from the global burden of disease 2013 study. ${ }^{30}$ From the Drugs@FDA database we extracted the date of the drug's approval, the date of the first label containing pharmacogenomic information, the first indication for the drug and, if different, the first indication for the drug with pharmacogenomic labeling, and the date the drug's sponsor submitted the application to the regulation agency, as previously described. ${ }^{5}$ From the European Medicines Agency (EMA) website we obtained the date of marketing authorization valid throughout the European Union and the date of submission of the application to the agency. We considered the first submission of an application to the FDA or EMA as a proxy for the end of drug development, and the first approval of the drug by one agency as the start of drug marketing. Trials with a start date before the first submission of an application were classified as "before submission," trials with a start date after the drug's approval by the FDA or EMA as "after approval," and trials in between as "between submission and approval."

\section{Statistical analysis}

We used only descriptive statistics. Continuous variables are presented as median and interquartile range because most variables are not normally distributed; qualitative variables are presented as frequencies (percentages). Analyses were performed with R version 3.1.0 (R Core Team, Vienna, Austria). 


\section{Included drugs and clinical trials}

The National Cancer Institute listed 69 oncology-targeted therapies; 22 also were included in the FDA Table of Pharmacogenomic Biomarkers in Drug Labeling and have a label requiring or recommending genetic testing. Nine drugs were approved before 2005 and were excluded. Everolimus was also excluded because it represented more than half of trials, and the majority corresponded to a new noncancer indication (prevention of organ rejection). Thus, this single drug may need a separate evaluation. We included 12 drugs (detailed characteristics are displayed in Supplementary Table S1 online). Overall, 935 studies corresponded to these drugs and were registered in ClinicalTrials.gov; we excluded 77 studies that did not fulfill inclusion criteria and therefore included 858 clinical trials (Supplementary Figure S1 online).

\section{Clinical study characteristics}

Characteristics of the 858 included studies are in Table 1. Half of the studies $(n=434,51 \%)$ were enriched on the first biomarker mentioned in the drug label and 145 (17\%) on another biomarker; 270 (31\%) included patients without restriction for a pharmacogenomic biomarker. Industry was the lead sponsor for 334 studies (39\%). The median sample size was 65 patients (interquartile range $(\mathrm{Q} 1-\mathrm{Q} 3)=35-154)$. The most frequent primary outcomes were response rate (37\%), event-free survival (19\%), and toxicity/dose finding (15\%). Most studies (72\%) started after the first FDA approval, and only $4 \%$ ended before approval. Overall, 497 studies (58\%) assessed drug effects for the same disease as the first approval. More than half of the studies (60\%) were single-group studies. For all drugs, the first biomarker was present at approval and corresponded to the drug's target. Figure 1 displays the number of trials and patients enrolled in enriched trials and in single-arm trials, by phase. Enriched trials as well as single-arm trials represented a large proportion of trials and of patients, especially phase III trials, which typically enrolled many patients.

\section{Mapping of clinical trials before and after FDA approval}

Figure 2 displays, for each drug, the number of trials by year and two characteristics of the trials: (i) the use of a biomarker to restrict inclusion in the trial (treat all patients, use of the same biomarker as the first one mentioned in the drug label, or use of another biomarker) and (ii) random assignment of treatment. Dates of first submission and first approval by the FDA or EMA (whichever came first) are shown by red and black vertical arrows, respectively. All drugs were tested in randomized trials that were restricted to the biomarker mentioned in the label (red areas). These trials could correspond to trials supporting drug approval and pharmacogenomic labeling. However, some drugs, such as afatinib, also were tested in nonenriched trials (green areas) and trials enriched on another biomarker (blue areas), whereas some drugs (e.g., dabrafenib) were tested almost exclusively in trials restricted to the biomarker mentioned in the label. ${ }^{21}$ Of note, the first trials of afatinib and pertuzumab were not enriched on any biomarker. Vemurafenib was the only drug tested in biomarker-negative patients before FDA approval. After FDA approval, some drugs (e.g., vemurafenib, ado-trastuzumab) were tested in only one disease, with trials enriched on the targeted biomarker. The most recent drugs (e.g., bosutinib, crizotinib) were obviously associated with a small number of trials after FDA approval. Dasatinib and nilotinib were associated with more trials of other diseases than approval indication. Crizotinib was tested in biomarker-negative patients after FDA approval, after a postmarketing commitment by the FDA in its medical review. ${ }^{31}$ Overall, among 237 trials started before FDA approval, 128 (54\%) were enriched on the biomarker first mentioned in the label and 27 (11\%) were enriched on another one, whereas among 614 trials started after FDA approval, 305 (50\%) were enriched on the first biomarker and 117 (19\%) on another biomarker.

\section{Evolution of biomarker and disease studied for each drug}

Table 1 also displays the main characteristics of clinical trials across three periods of drug development: (i) before the first submission to the FDA or EMA, (ii) after the first submission and before first approval by the FDA or EMA, and (iii) after approval. The share of industrysponsored trials dropped from $73 \%$ before submission to $28 \%$ after approval. The proportion of trials enriched on the label biomarker and for the corresponding indication ranged from $5 \%$ with panitumumab-treated EGFR-positive patients to $88 \%$ with ado-trastuzumab-treated HER-2-positive patients. This proportion was still high (sometimes higher) after drug approval.

Figure 3 shows hive plots representing the mapping of the number of clinical trials for drugs, biomarkers, and diseases. Multiple-disease trials are represented once for each studied disease, as are multiple-drug trials. Two patterns of drugs seem to emerge: The first corresponds to drugs tested almost solely in biomarker-positive patients and with the same disease first described on the label (ado-trastuzumab, dabrafenib, vemurafenib), and the second includes drugs tested in trials enriched on other biomarkers, in treat-all trials, or in other diseases (all other drugs). These patterns could be seen especially for recent drugs (e.g., ado-trastuzumab versus crizotinib). Among the first group, however, dabrafenib and vemurafenib were tested with some other diseases, but the biomarkers used to restrict trials were not expanded. In the second group, dasatinib, lapatinib, and nilotinib seem to be associated with the most diseases and biomarkers used in trials, resulting in very dense figures. Afatinib and bosutinib were tested with many diseases but, compared with the drugs in the second group, with a relatively small number of biomarkers. Among the 361 trials testing a drug for a disease different than the first indication, there was no enrichment for 219 trials (61\%); there was enrichment on the biomarker described in the label for 48 trials (13\%), enrichment on a different biomarker for 87 trials (24\%), and enrichment on multiple biomarkers for 7 trials (2\%). 
Table 1 Main characteristics of included studies, overall and stratified by phase of drug development

\begin{tabular}{|c|c|c|c|c|}
\hline & Overall & $\begin{array}{l}\text { Phase I: before } \\
\text { submission }\end{array}$ & $\begin{array}{l}\text { Phase II: between } \\
\text { submission and approval }\end{array}$ & $\begin{array}{l}\text { Phase III: after } \\
\text { approval }\end{array}$ \\
\hline Trials $^{a}$ & $858(100)$ & $182(21.2)$ & $55(6.4)$ & $614(71.6)$ \\
\hline \multicolumn{5}{|l|}{ Phase } \\
\hline$|/| \mid$ & $116(14)$ & $15(8)$ & $4(7)$ & $97(16)$ \\
\hline$\|$ & $581(68)$ & $127(70)$ & $39(71)$ & $409(67)$ \\
\hline III & $161(19)$ & $40(22)$ & $12(22)$ & $108(18)$ \\
\hline \multicolumn{5}{|l|}{ PGx used for enrichment } \\
\hline No PGx & $270(31)$ & $65(36)$ & $15(27)$ & $185(30)$ \\
\hline Same as label PGx & $434(51)$ & $93(51)$ & $35(64)$ & $305(50)$ \\
\hline Other than label PGx & $145(17)$ & $23(13)$ & $4(7)$ & $117(19)$ \\
\hline Multiple PGx & $9(1)$ & $1(1)$ & $1(2)$ & $7(1)$ \\
\hline Same disease as first on label & $497(58)$ & $105(58)$ & $33(60)$ & $357(58)$ \\
\hline \multicolumn{5}{|c|}{ Proportion of trials enriched on the label PGx and in the indication, no./total ${ }^{a}(\%)$} \\
\hline Ado-trastuzumab & $22 / 25(88)$ & $9 / 10(90)$ & $2 / 4(50)$ & $11 / 11(100)$ \\
\hline Afatinib & $13 / 59(22)$ & $6 / 39(15)$ & $2 / 9(22)$ & $5 / 11(45)$ \\
\hline Bosutinib & $5 / 11(45)$ & $3 / 9(33)$ & $0 / 0(0)$ & $2 / 2(100)$ \\
\hline Crizotinib & $9 / 20(45)$ & $3 / 5(60)$ & $0 / 0(0)$ & $6 / 15(40)$ \\
\hline Dabrafenib & $27 / 34(79)$ & $5 / 7(71)$ & $6 / 8(75)$ & $16 / 19(84)$ \\
\hline Dasatinib & $48 / 147(33)$ & $11 / 12(92)$ & $1 / 3(33)$ & $35 / 130(27)$ \\
\hline Lapatinib & $107 / 186(58)$ & $19 / 45(42)$ & $3 / 8(38)$ & $85 / 133(64)$ \\
\hline Nilotinib & $60 / 95(63)$ & $4 / 5(80)$ & $4 / 5(80)$ & $52 / 85(61)$ \\
\hline Panitumumab & $7 / 134(5)$ & $6 / 14(43)$ & $1 / 5(20)$ & $0 / 112(0)$ \\
\hline Pertuzumab & $35 / 55(64)$ & $12 / 23(52)$ & $2 / 3(67)$ & $21 / 27(78)$ \\
\hline Trametinib & $21 / 50(42)$ & $4 / 11(36)$ & $4 / 8(50)$ & $13 / 31(42)$ \\
\hline Vemurafenib & $32 / 42(76)$ & $2 / 2(100)$ & $1 / 2(50)$ & $29 / 38(76)$ \\
\hline Sample size, median (IQR) & $65(35-154)$ & $110(49-328)$ & $71(40-249)$ & $60(32-129)$ \\
\hline Trial duration (years), median (IQR) & $4.0(2.8-5.9)$ & $4.4(2.8-6.5)$ & $3.8(2.7-4.8)$ & $4.0(2.8-5.8)$ \\
\hline \multicolumn{5}{|l|}{ Sponsor } \\
\hline Industry & $334(39)$ & $133(73)$ & $27(49)$ & $171(28)$ \\
\hline National Institutes of Health & $63(7)$ & $18(10)$ & $2(4)$ & $43(7)$ \\
\hline Other & $461(54)$ & $31(17)$ & $26(47)$ & $400(65)$ \\
\hline \multicolumn{5}{|l|}{ Allocation } \\
\hline Missing & $13(2)$ & $7(4)$ & $1(2)$ & $3(0)$ \\
\hline Nonrandomized & $551(64)$ & $108(59)$ & $29(53)$ & $410(67)$ \\
\hline Randomized & $294(34)$ & $67(37)$ & $25(45)$ & $201(33)$ \\
\hline \multicolumn{5}{|l|}{ Design } \\
\hline Controlled & 334 (39) & $79(43)$ & $30(55)$ & $223(36)$ \\
\hline Single-arm & $513(60)$ & $98(54)$ & $25(45)$ & $386(63)$ \\
\hline Missing & $11(1)$ & $5(3)$ & $0(0)$ & $5(1)$ \\
\hline \multicolumn{5}{|l|}{ Masking } \\
\hline Missing & $12(1)$ & $4(2)$ & $0(0)$ & $7(1)$ \\
\hline Double blind & $48(6)$ & $15(8)$ & $5(9)$ & $28(5)$ \\
\hline Open label & $796(93)$ & $162(89)$ & $50(91)$ & $578(94)$ \\
\hline Single blind & $2(0)$ & $1(1)$ & $0(0)$ & $1(0)$ \\
\hline \multicolumn{5}{|l|}{ Type of study } \\
\hline Efficacy & $216(25)$ & $32(18)$ & $10(18)$ & $174(28)$ \\
\hline Pharmacology & $10(1)$ & $3(2)$ & $3(6)$ & $4(1)$ \\
\hline Safety & $34(4)$ & $3(2)$ & $3(5)$ & $27(4)$ \\
\hline Safety/efficacy & $502(59)$ & $120(66)$ & $34(62)$ & $346(56)$ \\
\hline Missing & $96(11)$ & $24(13)$ & $5(9)$ & $63(10)$ \\
\hline \multicolumn{5}{|l|}{ Primary outcome } \\
\hline Event-free survival & $162(19)$ & $40(22)$ & $10(18)$ & $111(18)$ \\
\hline Other/unclassified & $172(20)$ & $24(13)$ & $14(25)$ & $130(21)$ \\
\hline Overall survival & $73(9)$ & $14(8)$ & $3(5)$ & $56(9)$ \\
\hline Response rate & $319(37)$ & $84(46)$ & $19(35)$ & $215(35)$ \\
\hline Time to progression & $4(0)$ & $1(1)$ & $0(0)$ & $3(0)$ \\
\hline Toxicity/dose finding & $128(15)$ & $19(10)$ & $9(16)$ & $99(16)$ \\
\hline
\end{tabular}

Data are no. (\%) unless otherwise specified.

IQR, interquartile range; $\mathrm{PGX}$, pharmacogenomic biomarker.

aData for the start date of the trial were missing for seven studies. 

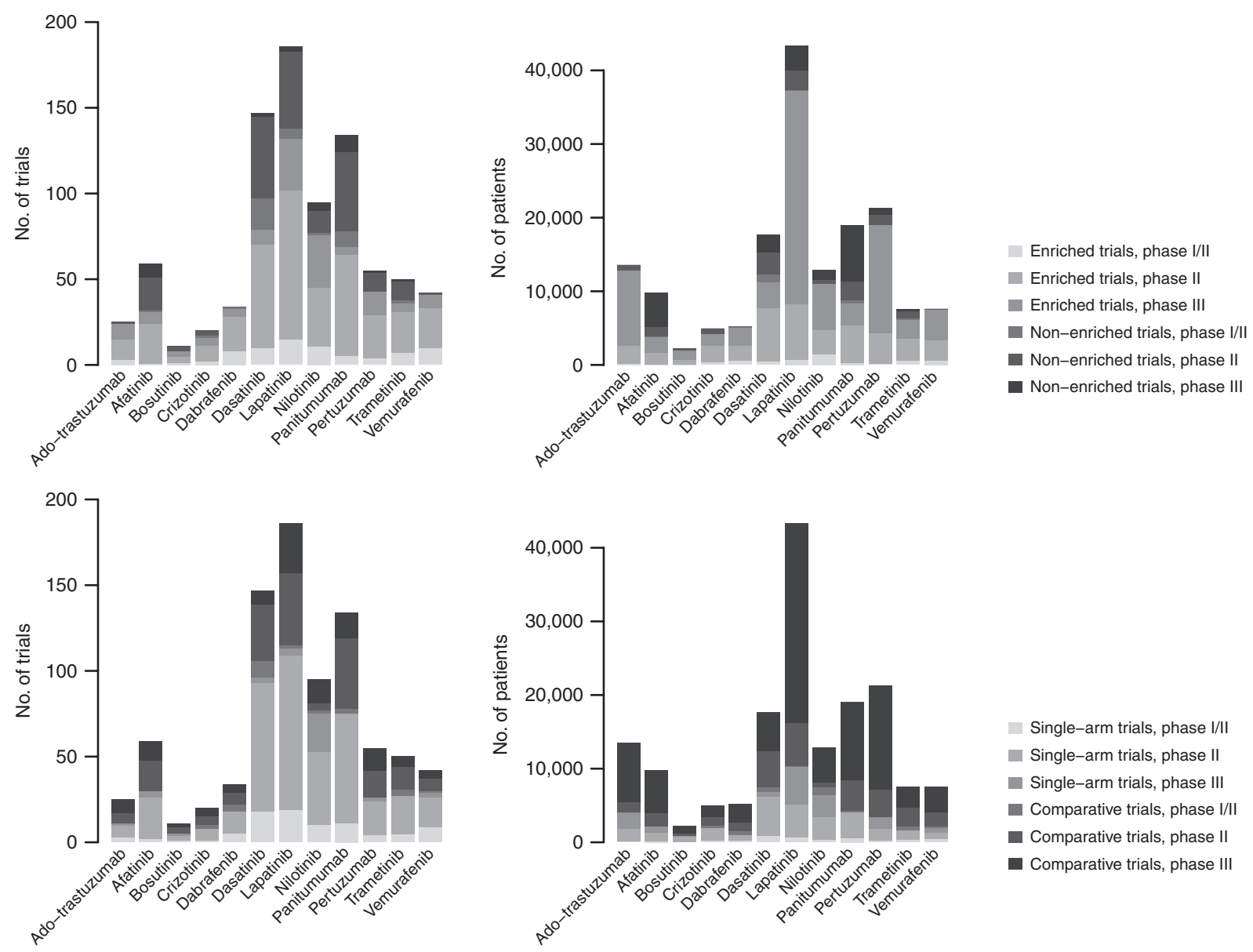

Figure 1 Number of trials (left) and of patients (right) enrolled in trials registered on ClinicalTrials.gov, by drug, 2005-2014. The top panel contrasts enriched trials (light) versus nonenriched trials (dark). The bottom panel contrasts single-arm trials versus comparative trials.

\section{DISCUSSION}

This mapping of clinical trials of targeted drugs with biomarker labeling shows a great diversity in the development of targeted drugs. Indeed, some targeted therapies have been tested with only one biomarker (which is the drug target) and in only one disease, whereas others drugs have been tested with several biomarkers (which are not the drug's target) and in several diseases. Another finding of this study is that, although the efficacy of some drugs was studied both in biomarker-positive and -negative patients, most trials were enriched single-arm trials, and several drugs were developed with only biomarker-positive patients included in trials. For those drugs, we therefore have no formal clinical evidence for restricting treatment to biomarker-positive patients.

The rationale to test or not test a targeted therapy in patients other than the ones who are positive for the target biomarker (i.e., biomarker-negative patients or patients who are positive for another biomarker) depends on the strength of preclinical data and the rationale for drug targeting, ${ }^{9,12}$ the relevance of which is not discussed here. However, this point deserves careful consideration because of the risk of restricting drug indications to the wrong subpopulation-as has initially been the case for cetuximab and EGFR expression before the predictive role of RAS mutations were shown; this needs to be balanced against the ethical dilemma of exposing biomarkernegative patients to a drug from which one thinks they will not derive benefit, and strategic consideration of drug development from the drug's sponsor. Moreover, enrichment-only drug development reduces the number of patients exposed to the drug and therefore may also hinder the validity of drug safety evaluation. However, compared with the number of investigational drugs to be tested in clinical trials, in some therapeutic areas only a few patients may be eligible; thus, restricting trials to biomarker-positive patients could allow for biomarker-negative patients to be included in another trial that is more likely to be beneficial for them. Of note, at least one health authority agency (the French Haute Autorité de Santé) considered a drug to be targeted only with a high level of evidence of qualitative treatment-marker interaction (i.e., the treatment is effective only in biomarker-positive patients) and recommended a randomize-all design with randomization stratified by biomarker results. ${ }^{9}$

Nearly one-quarter (27\%) of enriched trials used a biomarker other than the first one mentioned on the drug's label. For example, trametinib, a mitogen-activated protein kinase (MEK) inhibitor, also has been studied in trials restricted to 

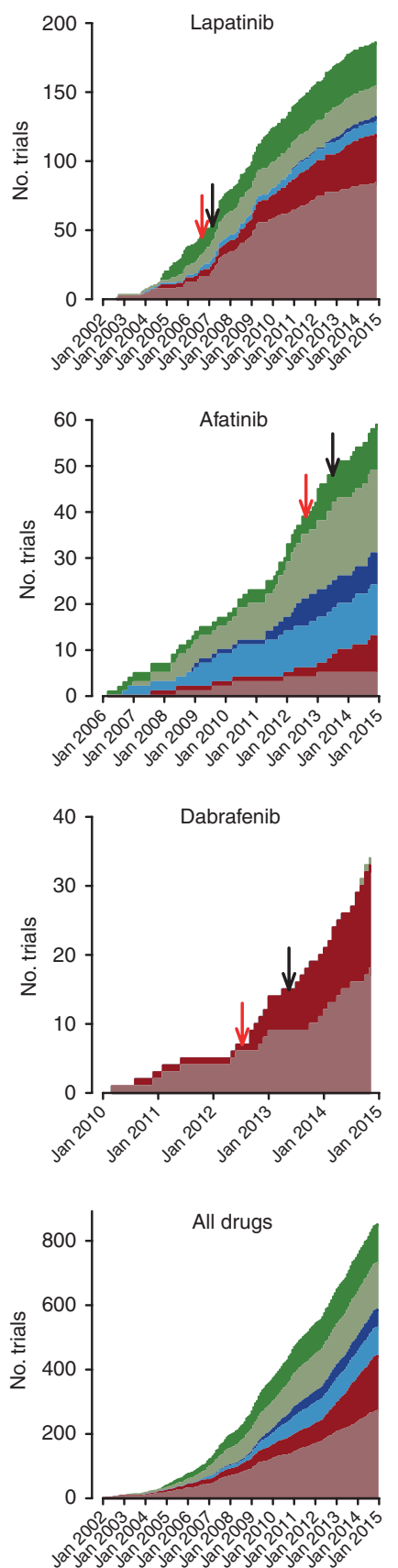
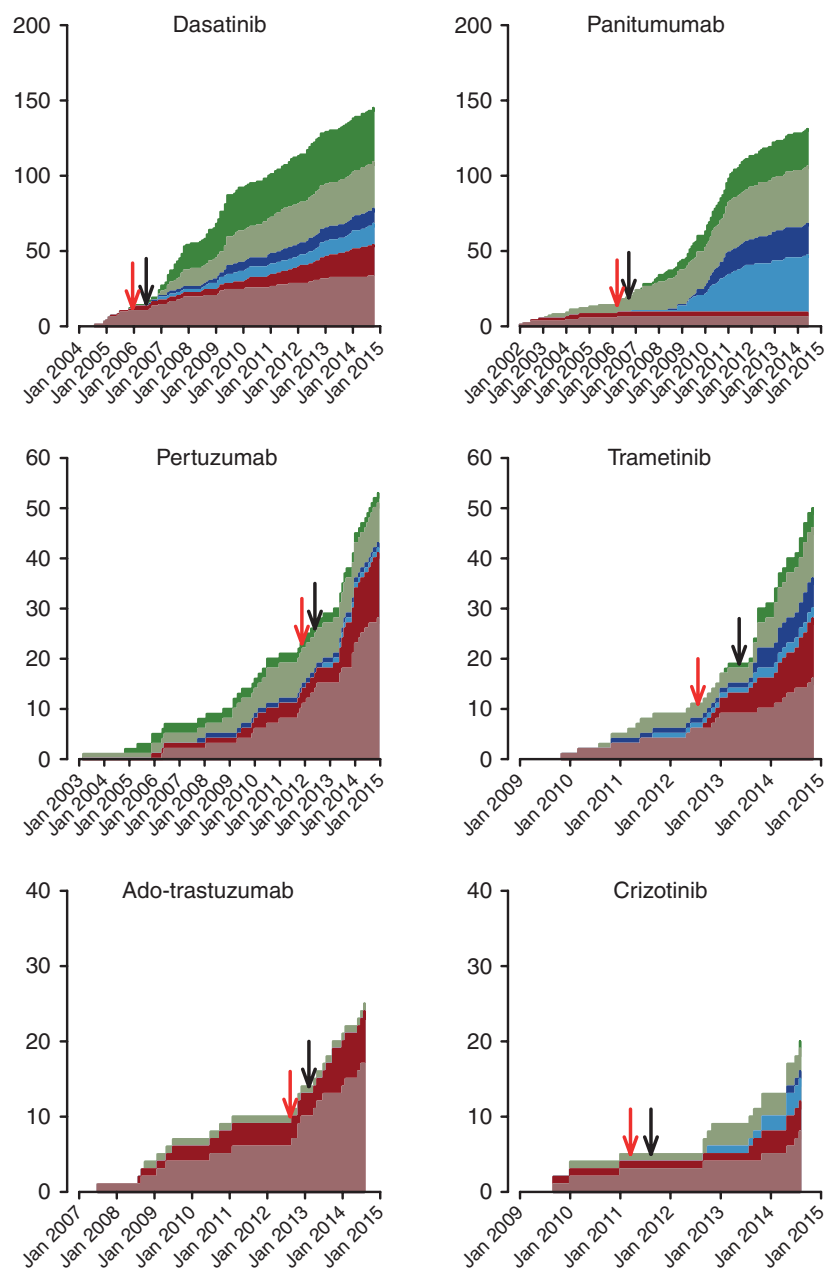

Trials enriched on the labeled PGx, randomized

Trials enriched on the labeled PGx non-randomized

$\square$ Trials enriched on another PGx, non-randomized

$\square$ Non-enriched trials, non-randomized randomized

$\square$ Non-enriched trials, randomized
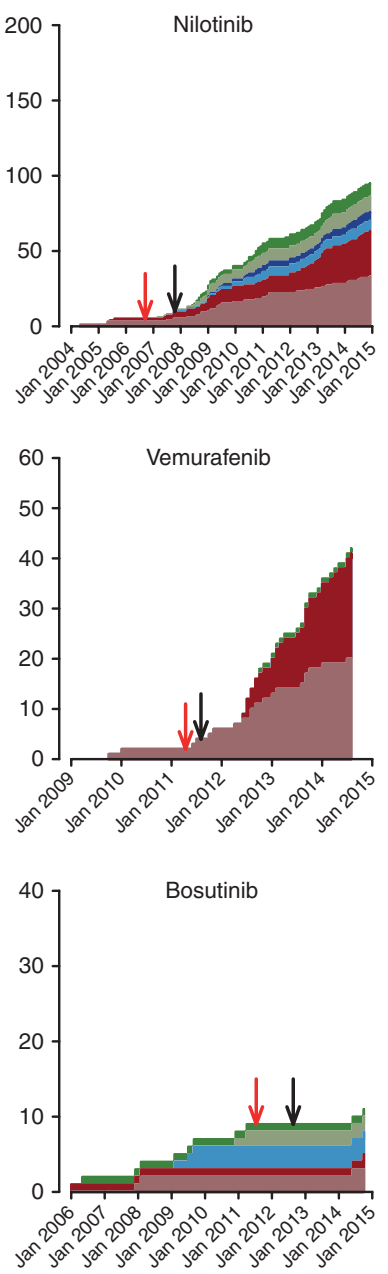

Date of first submission

Date of first approval

Figure 2 Cumulative number of trials registered on ClinicalTrials.gov, by calendar time, 2005-2014. The cumulative number of trials is stratified by use of enrichment design and randomization regrouped into six categories: nonrandomized and randomized trials enriched on the same pharmacogenomics as mentioned in the label (dark and light red, respectively), nonrandomized and randomized trials enriched on another pharmacogenomic (dark and light blue, respectively), and nonrandomized and randomized nonenriched trials (dark and light green, respectively). Red areas represent trials that are restricted to the biomarker mentioned in the label; blue, trials enriched on another biomarker; and green, nonenriched trials. Lighter areas represent randomized trials, darker areas represent nonrandomized trials. The charts are sorted by number of trials. Vertical axes go up to 200 for the first row, 60 for the second, 40 for the third, and 800 for the last row. Dates of first submission and first approval by the US Food and Drug Administration (FDA) or European Medicines Agency (EMA) are shown by red and black vertical arrows, respectively.

patients with KRAS mutations or HER-2 expression. Similarly, afatinib has been studied in several trials of HER-2-positive tumors, although its first biomarker is EGFR. However, EGFR and HER-2 are both part of the ErbB family (ErbB-1 and ErbB2 , respectively), and afatinib has an affinity for HER-2. There are several reasons to test a drug with biomarker other than the first one. First, several postapproval studies have attempted to discover response/resistance biomarkers, and some of these are currently in the clinical validation phase. Second, some included drugs are highly target-selective, such as dabrafenib (which is a mutant BRAF V600-specific inhibitor), but other drugs do have affinity for targets other than their initial ones. 


\section{ORIGINAL RESEARCH ARTICLE}

This could explain the choice of biomarkers in some of those trials, such as crizotinib in ROS proto-oncogene 1, receptor tyrosine kinase (ROS-1)-positive patients, dasatinib in plateletderived growth factor receptor-positive patients, or nilotinib in v-kit Hardy-Zuckerman 4 feline sarcoma viral oncogene homo$\log (\mathrm{KIT})$-positive and platelet-derived growth factor receptor B-positive patients.

a
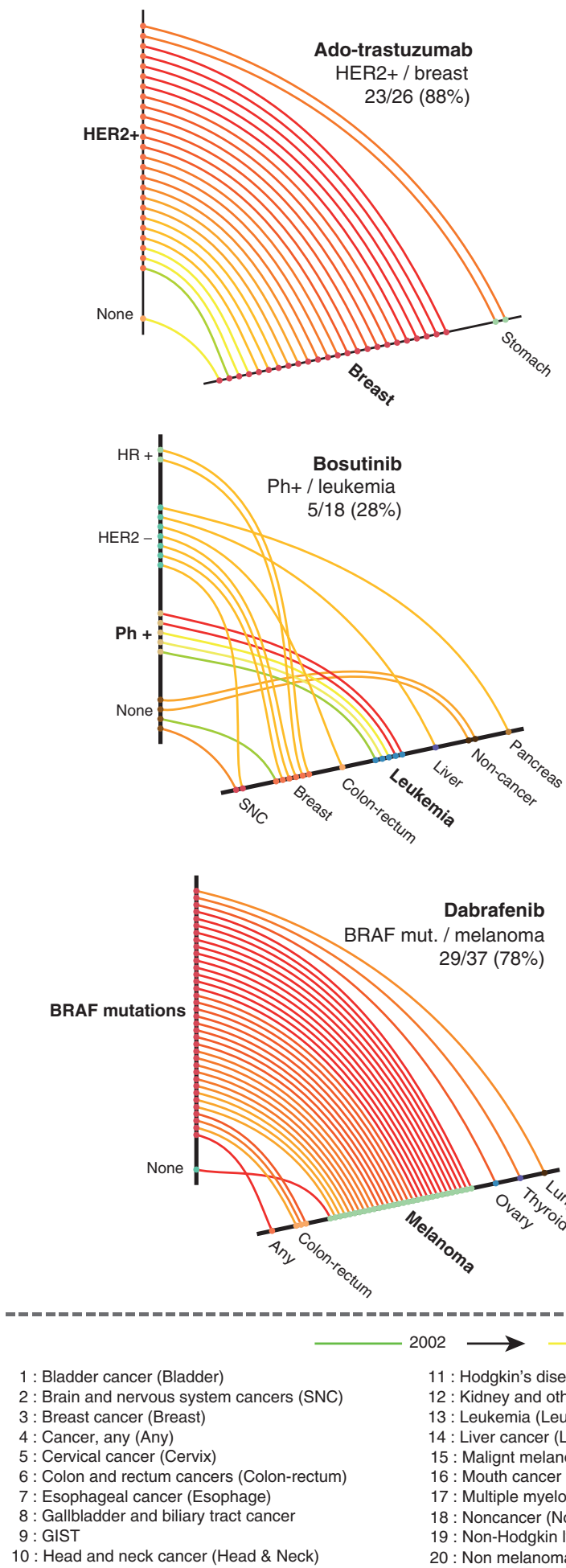

Dabrafenib BRAF mut. / melanoma $29 / 37$ (78\%)

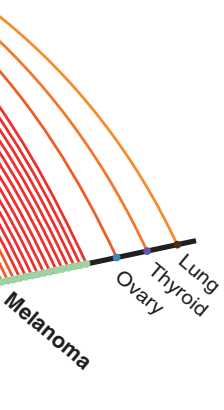

Another likely explanation of the differences in development schemes could be the differences between drugs developed to target a specific mutation (dasatinib, nilotinib, afatinib, bosutinib, crizotinib, dabrafenib, vemurafenib, and trametinib) and drugs developed to target a molecular pathway (lapatinib, panitumumab, pertuzumab, ado-trastuzumab). Development may be more targeted for the former group than for the latter.
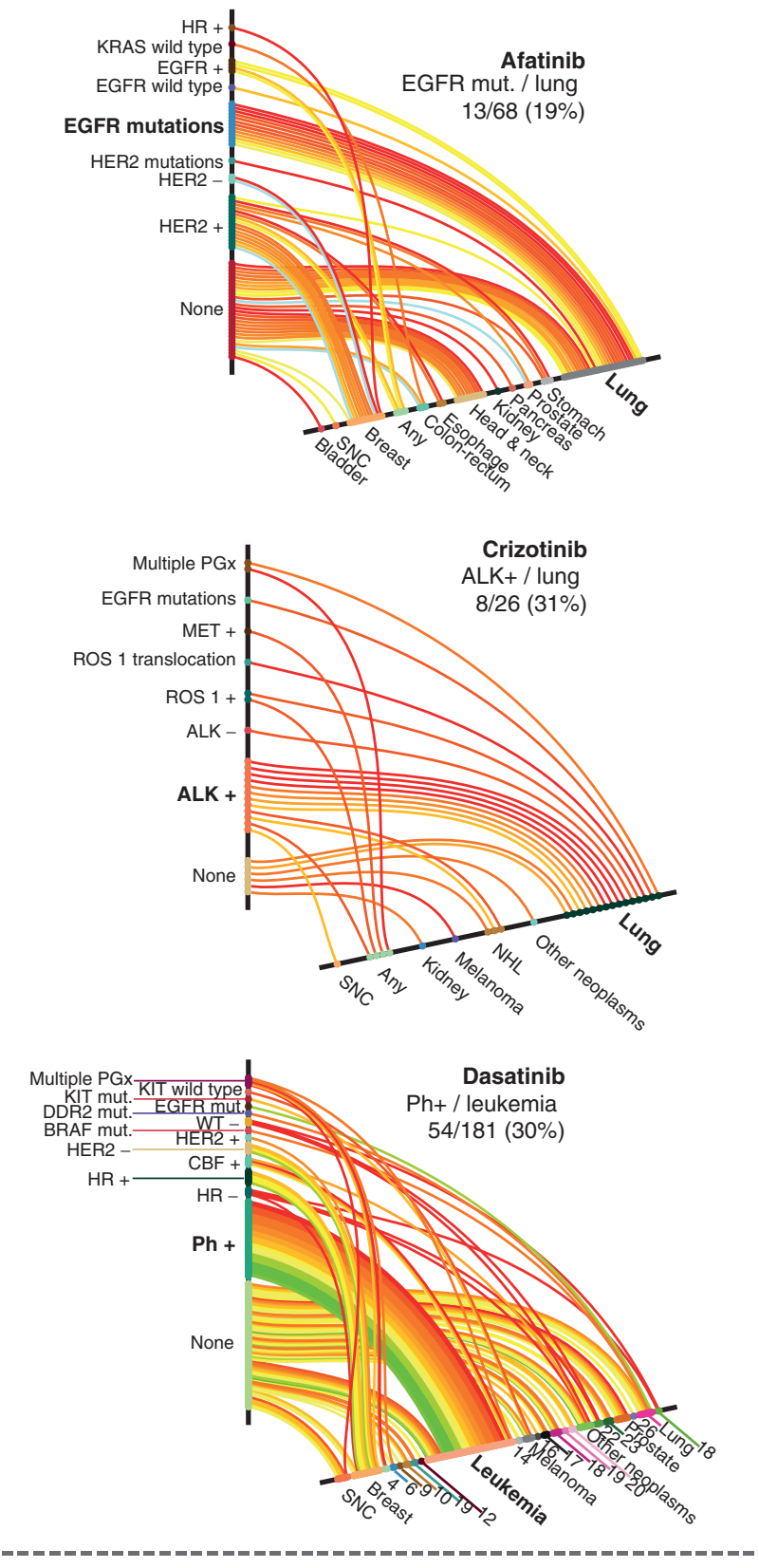

$2002 \longrightarrow \longrightarrow$
$11:$ Hodgkin's disease (Hodgkin)
$12:$ Kidney and other uriry organ cancers (Kidney)
$13:$ Leukemia (Leukemia)
$14:$ Liver cancer (Liver)
$15:$ Malignt melanoma of skin (Melanoma)
$16:$ Mouth cancer (Mouth)
$17:$ Multiple myeloma (Myeloma)
$18:$ Noncancer (Noncancer)
$19:$ Non-Hodgkin lymphoma (NHL)
$20:$ Non melanoma skin cancer (Skin)

2014

21 : Other neoplasms (Other neoplasms)

22 : Ovarian cancer (Ovary)

23 : Pancreatic cancer (Pancreas)

24 : Prostate cancer (Prostate)

25 : Stomach cancer (Stomach)

26 : Testicular cancer (Testicular)

27 : Thyroid cancer (Thyroid)

28 : Trachea, bronchus, and lung cancers (Lung)

29 : Uterine cancer (Uterus) 


\section{b}
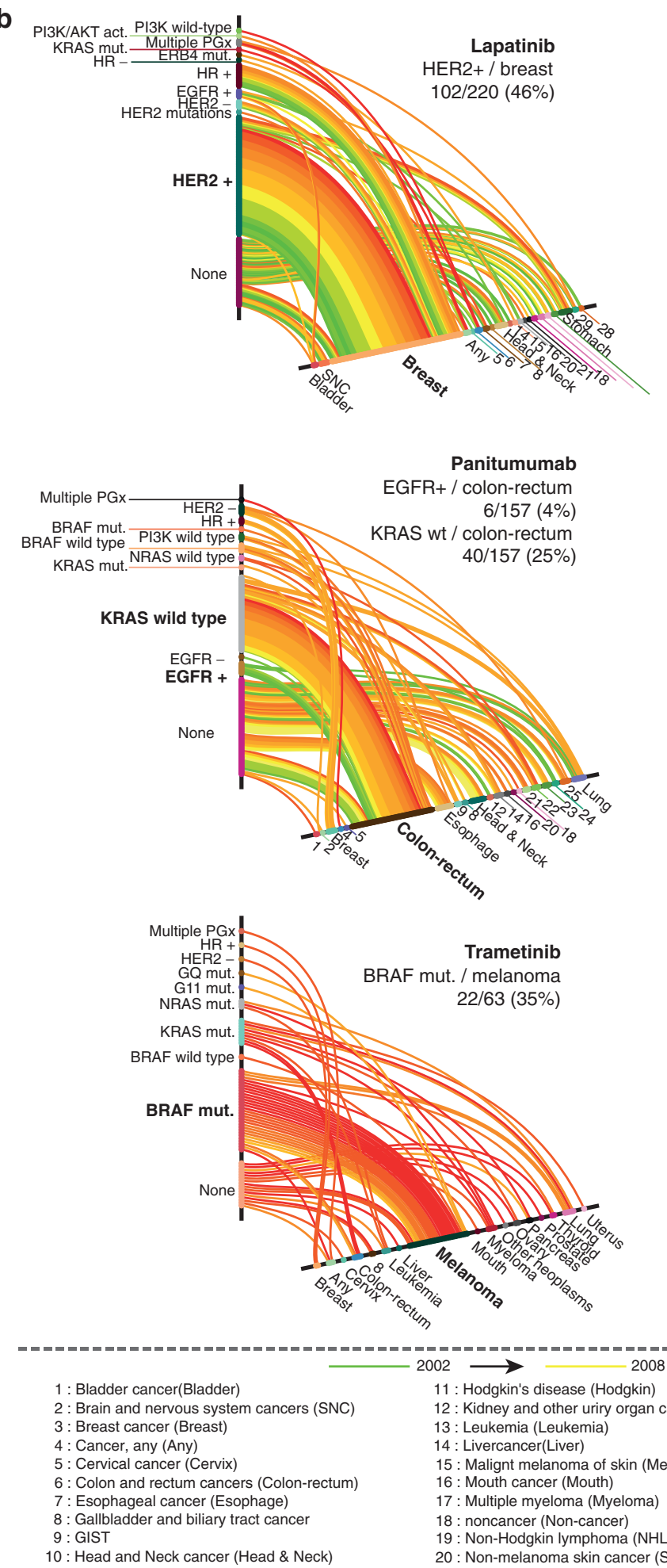
Trametinib $22 / 63(35 \%)$
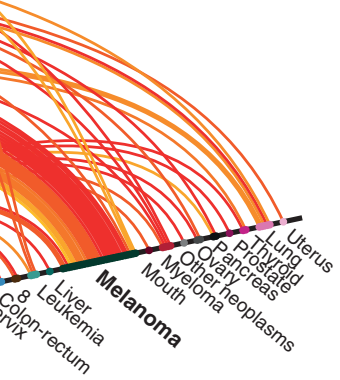

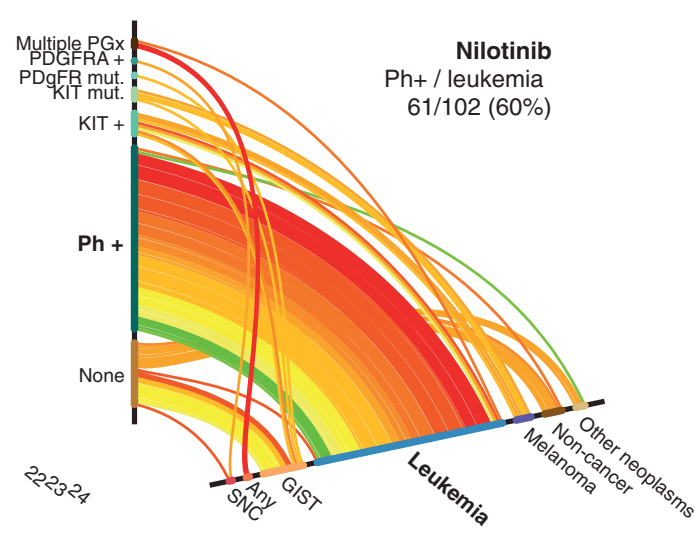

Pertuzumab
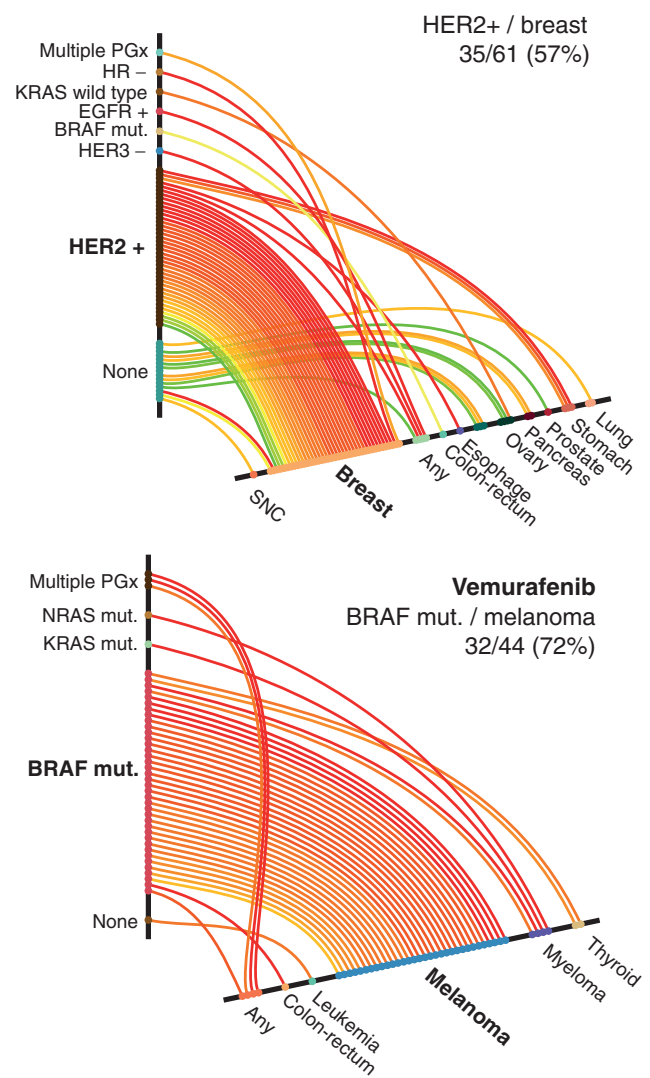

$\begin{array}{ll}\text { 11: Hodgkin's disease (Hodgkin) } & 21: \text { Other neoplasms (Other neoplasms) } \\ 12: \text { Kidney and other uriry organ cancers (Kidney) } & 22: \text { Ovarian cancer (Ovary) } \\ 13: \text { Leukemia (Leukemia) } & 23: \text { Pancreatic cancer (Pancreas) } \\ \text { 14: Livercancer(Liver) } & 24: \text { Prostate cancer (Prostate) } \\ 15: \text { Malignt melanoma of skin (Melanoma) } & 25: \text { Stomach cancer (Stomach) } \\ 16: \text { Mouth cancer (Mouth) } & 26: \text { Testicular cancer (Testicular) } \\ 17: \text { Multiple myeloma (Myeloma) } & 27: \text { Thyroid cancer (Thyroid) } \\ 18: \text { noncancer (Non-cancer) } & 28: \text { Trachea, bronchus, and lung cancers (Lung) } \\ 19: \text { Non-Hodgkin lymphoma (NHL) } & 29: \text { Uterine cancer (Uterus) }\end{array}$

Figure 3 Graphical representation (hive plots) of the disease-biomarker network in trials registered on ClinicalTrials.gov. (a) Hive plots for Ado-trastuzumab, Afatinib, Bosutinib, Crizotinib, Dabrafenib, and Dasatinib. (b) Hive plots for Lapatinib, Nilotinib, Panitumumab, Pertuzumab, Trametinib and Vemurafenib. Each hive plot represents a targeted therapy; within each plot, each trial is represented by a link between the biomarker used to restrict inclusion in the trial (vertical axis) and the disease studied (horizontal axis). The color of the link corresponds to the start year of the trial: green represents trials from 2002; yellow, studies from 2008; and red, trials from 2014; intermediate dates are represented by gradients between colors. Trials with a treat-all design are linked to the group "None" in the vertical axis. Multiple-disease trials are represented once for each studied disease, and multiple-drug trials are represented once for each studied drug. Biomarker-indication pairs for which genetic testing is recommended or required are in bold and indicated below the drug name, along with the proportion of corresponding trials. 


\section{ORIGINAL RESEARCH ARTICLE}

However, dabrafenib and vemurafenib both displayed a targeted development scheme (i.e., a small network), whereas trametinib displayed a much less targeted development scheme (i.e., a wider network), although these three drugs were developed to target $B R A F$ V600 mutations. Overall, the disease-biomarker pairs were similar across drugs, so the disease-biomarker association may be more specific than the drug-biomarker association. Furthermore, several trials involved multiple biomarkers. These trials could test multiple biomarkers in one disease or with one drug, such as the AcSé (NCT02034981) or CREATE (Crosstumoral Phase 2 With Crizotinib, NCT01524926) trials, and are called "basket" trials. ${ }^{32}$ By contrast, "umbrella" trials such as NCIMPACT $^{33}$ or SHIVA ${ }^{34}$ tested multiple biomarkers across a broad range of tumor types. ${ }^{35}$ In the open-label, controlled, phase II SHIVA trial, patients with metastatic solid tumor refractory to the standard of care and with a molecular alteration in phosphoinositide 3-kinase/AKT/mammalian target of rapamycin, $\mathrm{RAF} / \mathrm{MEK}$, or hormone receptor pathways were randomized to matched molecularly targeted agents in a histology-agnostic way and the physician's choice of treatment. There were no differences in primary outcome (progression-free survival) between the two groups: median progression-free survival was 2.3 months (95\% confidence interval: 1.7-3.8) in the experimental group versus 2.0 months (1.8-2.1) in the control group (hazard ratio $0.88 ; 95 \%$ confidence interval: $0.65-1.19 ; P=0.41) .{ }^{34}$ These results suggest that relying solely on biomarker (i.e., without histology) to predict efficacy may not yet be possible in oncology.

Our results are consistent with a previous study of general oncology trials, finding that, compared with nononcology trials, oncology trials were more often single-arm, open-label, and nonrandomized, ${ }^{36}$ a pattern we also found for trials of targeted therapies. We did not specifically assess the characteristics of pivotal trials in this study, but for the 55 oncology novel therapeutic agents approved by the FDA between 2005 and 2012, only half of the pivotal trials were randomized, $27 \%$ were double-blind, and only $16 \%$ had a clinical outcome as a primary end point, reflecting a lower level of evidence of studies for FDA approval in oncology compared with nononcology areas..$^{37}$ This finding could be explained in part by the high frequency of orphan drugs in oncology and the lower level of evidence for such drugs compared with nonorphan oncology drugs. ${ }^{38}$ This relatively low level of evidence of trials could also be explained by the high number of indications (either the first or subsequent ones) approved under the accelerated approval process; pivotal studies supporting accelerated approvals are often early-phase, nonrandomized trials using surrogate end points. ${ }^{36,37} \mathrm{~A}$ study of pharmacogenomic information in drug labels approved by the EMA found that two-thirds of pivotal trials for drugs with a biomarker-based indication have been conducted in only biomarker-positive patients, ${ }^{39}$ a pattern we also found in our study of all (not only pivotal) trials.

One strength of this study is the use of data from ClinicalTrials. gov. Because clinical trials take a long time to complete, studies registered in clinical trial registries can provide information before they end, and therefore provide a preview of which studies are currently ongoing. However, the drawback is that there is no access to the results of those trials. This study has several limitations. We used only US data (labels from the FDA and trials registered at ClinicalTrials.gov) because we made the assumption that a US-centric view represents biomarker usage in trials of targeted therapies. Some trials may have been registered in registry other than ClinicalTrials.gov, but this would concern only trials conducted outside the United States; publication in ClinicalTrials. gov is mandatory for trials with at least one center in the United States. Some trials may have been conducted before trial registration was required; thus we excluded drugs approved before 2005. We analyzed data as provided by the trial's sponsor, which may suggest some errors in coding when the study was registered. Furthermore, we analyzed only targeted therapies with biomarker-based indications because we were interested in how targeted drugs that are restricted to biomarker-positive patients have been developed and especially if the development was targeted and how this might evolve after drug approval. Studying the use of biomarkers in clinical trials of targeted therapies without biomarker-based indication warrants further investigation.

\section{Conclusion}

Oncology-targeted therapies have undoubtedly set the stage for precision medicine, but the drug-biomarker-disease network is actually more complex than it would seem at first glance. The rationale to test or not test a targeted therapy in patients other than the ones who are positive for the target biomarker (i.e., biomarker-negative patients or patients who are positive for another biomarker) deserves careful consideration because of the risk of over-restricting drug indications and of exposing patients to a drug from which they are not likely to derive benefit.

\section{SUPPLEMENTARY MATERIAL}

Supplementary material is linked to the online version of the paper at http://www.nature.com/gim

\section{ACKNOWLEDGMENTS}

A.V. is supported by a French grant "Soutien pour la formation à la recherche translationnelle en cancérologie, édition 2014" with financial support from ITMO Cancer AVIESAN (Alliance Nationale pour les Sciences de la Vie et de la Santé, National Alliance for Life Sciences and Health) within the framework of the Cancer Plan, 2014-2019. The authors thank Ignacio Atal (INSERM U1153, Paris, France) for providing the computer program to extract ClinicalTrials.gov data, Ludovic Trinquart (INSERM U1153, Paris, France) for suggesting the use of hive plots, Elise Diard (INSERM U1153, Paris, France) for help with artwork, and Laura Smales (BioMedEditing, Toronto, Canada) for proofreading.

The sponsors have no role in study design, data collection and analysis, decision to publish, or preparation of the manuscript.

\section{DISCLOSURE}

J.-D.Z. reports that he serves as an adviser for several consulting firms and communication companies linked to the pharmaceutical 
industry (Cepton, Oliver Wyman, McCann Healthcare, Omnicom, Canal 55, Grey Healthcare, Saatchi and Saatchi Healthcare, Sudler \& Hennessey, TBWA, inVentiv Health France, Havas). He also reports collaboration with Mayoly-Spindler, Merck, Teva, and Menarini; unpaid consultancy for EY and Allurion Technologies; conducting workshops funded by Amgen; and being invited to a French medical congress by AbbVie. He is an in-house adviser at The Family, an investment company located in London and Paris, where he is responsible for start-ups dedicated to health care. S.M. reports membership on the scientific committee boards of Roche, Novartis, and Janssen. The other authors declare no conflict of interest.

\section{REFERENCES}

1. de Bono JS, Ashworth A. Translating cancer research into targeted therapeutics. Nature 2010;467:543-549.

2. Schott $A F$, Perou $C M$, Hayes DF. Genome medicine in cancer: What's in a name? Cancer Res 2015;75:1930-1935.

3. Gonzalez de Castro D, Clarke PA, Al-Lazikani B, Workman P. Personalized cancer medicine: molecular diagnostics, predictive biomarkers, and drug resistance. Clin Pharmacol Ther 2013:93:252-259.

4. Stegmeier F, Warmuth M, Sellers WR, Dorsch M. Targeted cancer therapies in the twenty-first century: lessons from imatinib. Clin Pharmacol Ther 2010;87:543-552.

5. Vivot A, Boutron I, Ravaud P, Porcher R. Guidance for pharmacogenomic biomarker testing in labels of FDA-approved drugs. Genet Med 2015;17:733-738.

6. US Food and Drug Administration. Guidance for Industry. Enrichment Strategies for Clinical Trials to Support Approval of Human Drugs and Biological Products. http://www.fda.gov/downloads/Drugs/ GuidanceComplianceRegulatoryInformation/Guidances/UCM332181.pdf. Accessed 18 March, 2015.

7. Wang B, Canestaro WJ, Choudhry NK. Clinical evidence supporting pharmacogenomic biomarker testing provided in US Food and Drug Administration drug labels. JAMA Intern Med 2014;174:1938-1944.

8. Simon R, Blumenthal GM, Rothenberg ML, et al. The role of nonrandomized trials in the evaluation of oncology drugs. Clin Pharmacol Ther 2015;97:502-507.

9. Haute autorité de santé. Companion diagnostic test associated with a targeted therapy: scientific appendix. April 2014. http://www.has-sante.fr/portail/ jcms/c_1776662/en/companion-diagnostic-test-associated-with-a-targetedtherapy-scientific-appendix. Accessed 2 March, 2015.

10. European Medicines Agency. Reflection paper on methodological issues associated with pharmacogenomic biomarkers in relation to clinical development and patient selection. June 2011. http://www. ema.europa.eu/ema/pages/includes/document/open_document. jsp?webContentld=WC500108672.13 March, 2015.

11. Temple R. Enrichment of clinical study populations. Clin Pharmacol Ther 2010;88:774-778.

12. Sleijfer S, Bogaerts J, Siu LL. Designing transformative clinical trials in the cancer genome era. J Clin Oncol 2013;31:1834-1841.

13. Messersmith WA, Ahnen DJ. Targeting EGFR in colorectal cancer. N Eng/ J Med 2008;359:1834-1836.

14. Graf AC, Posch M, Koenig F. Adaptive designs for subpopulation analysis optimizing utility functions. Biom J 2015;57:76-89.

15. Paik S, Kim C, Wolmark N. HER2 status and benefit from adjuvant trastuzumab in breast cancer. N Engl J Med 2008;358:1409-1411.

16. Ardavanis A, Kountourakis P, Kyriakou F, et al. Trastuzumab plus paclitaxel or docetaxel in HER-2-negative/HER-2 ECD-positive anthracycline- and taxanerefractory advanced breast cancer. Oncologist 2008;13:361-369.

17. Tuma RS. Cancer stem cell hypothesis and trastuzumab in HER2-negative tumors. J Natl Cancer Inst 2012;104:968-969.

18. Chung KY, Shia J, Kemeny NE, et al. Cetuximab shows activity in colorectal cancer patients with tumors that do not express the epidermal growth factor receptor by immunohistochemistry. J Clin Oncol 2005;23: 1803-1810.

19. Phelps MA, Sparreboom A. A snapshot of challenges and solutions in cancer drug development and therapy. Clin Pharmacol Ther 2014;95:341-346.

20. Atreya CE, Corcoran RB, Kopetz S. Expanded RAS: refining the patient population. J Clin Oncol 2015;33:682-685.

21. Amado RG, Wolf $M$, Peeters $M$, et al. Wild-type KRAS is required for panitumumab efficacy in patients with metastatic colorectal cancer. J Clin Oncol 2008;26:1626-1634.

22. Fridlyand J, Simon RM, Walrath JC, et al. Considerations for the successful co-development of targeted cancer therapies and companion diagnostics. Nat Rev Drug Discov 2013;12:743-755.

23. Zineh I, Huang SM. Biomarkers in drug development and regulation: a paradigm for clinical implementation of personalized medicine. Biomark Med 2011;5:705-713

24. Parkinson DR, Johnson BE, Sledge GW. Making personalized cancer medicine a reality: challenges and opportunities in the development of biomarkers and companion diagnostics. Clin Cancer Res 2012;18:619-624.

25. Woodcock J. The prospects for "personalized medicine" in drug development and drug therapy. Clin Pharmacol Ther 2007;81:164-169.

26. National Cancer Institute. Targeted Cancer Therapies. http://www.cancer.gov/ cancertopics/factsheet/Therapy/targeted. Accessed 12 January 2015.

27. US Food and Drug Administration. Genomics-Table of Pharmacogenomic Biomarkers in Drug Labeling. http://www.fda.gov/drugs/scienceresearch/ researchareas/pharmacogenetics/ucm083378.htm. Accessed 20 June 2014.

28. Dickersin $\mathrm{K}$, Rennie $\mathrm{D}$. The evolution of trial registries and their use to assess the clinical trial enterprise. JAMA 2012;307:1861-1864.

29. Kurzrock R, Kantarjian HM, Druker BJ, Talpaz M. Philadelphia chromosomepositive leukemias: from basic mechanisms to molecular therapeutics. Ann Intern Med 2003:138:819-830.

30. GBD 2013 Mortality and Causes of Death Collaborators. Global, regional, and national age-sex specific all-cause and cause-specific mortality for 240 causes of death, 1990-2013: a systematic analysis for the Global Burden of Disease Study 2013. Lancet 2015;385:117-171.

31. US Food and Drug Administration. Center for drug evaluation and research. Application number: 2025700rig1s000. Medical review(s). August 2011. http://www.accessdata.fda.gov/drugsatfda_docs/ nda/2011/2025700rig1s000MedR.pdf.

32. Menis J, Hasan B, Besse B. New clinical research strategies in thoracic oncology: clinical trial design, adaptive, basket and umbrella trials, new end-points and new evaluations of response. Eur Respir Rev 2014;23:367-378.

33. Kummar S, Williams M, Lih C-J, et al. NCI MPACT: National Cancer Institute molecular profiling-based assignment of cancer therapy [abstract]. J Clin Oncol 2014;32:5s.

34. Tourneau CL, Delord J-P, Gonçalves A, et al. Molecularly targeted therapy based on tumour molecular profiling versus conventional therapy for advanced cancer (SHIVA): a multicentre, open-label, proof-of-concept, randomised, controlled phase 2 trial. Lancet Oncol 2015;16:1324-1334.

35. Herbst RS, Gandara DR, Hirsch FR, et al. Lung master protocol (Lung-MAP)—a biomarker-driven protocol for accelerating development of therapies for squamous cell lung cancer: SWOG S1400. Clin Cancer Res 2015;21:15141524.

36. Hirsch BR, Califf RM, Cheng SK, et al. Characteristics of oncology clinical trials: insights from a systematic analysis of ClinicalTrials.gov. JAMA Intern Med 2013;173:972-979.

37. Downing NS, Aminawung JA, Shah ND, Krumholz HM, Ross JS. Clinical trial evidence supporting FDA approval of novel therapeutic agents, 2005-2012. JAMA 2014;311:368-377.

38. Kesselheim AS, Myers JA, Avorn J. Characteristics of clinical trials to support approval of orphan vs nonorphan drugs for cancer. JAMA 2011;305:23202326.

39. Ehmann F, Caneva L, Prasad K, et al. Pharmacogenomic information in drug labels: European Medicines Agency perspective. Pharmacogenomics J 2015;15:201-210. 